\title{
LOS JUECES FRENTE AL “COTO VEDADO"
}

Roberto Gargarella (Universidad Di Tella)

\section{Introducción}

En Derechos y justicia procesal imperfecta, José Juan Moreso nos presenta, con la claridad y agudeza que caracterizan sus trabajos, valiosos argumentos relacionados con un debate que lleva décadas y que parece haberse revitalizado en los últimos años: se trata del debate acerca de cómo proteger los derechos fundamentales, y de cuál debe ser el rol de la justicia en el cumplimiento de esta función. ${ }^{1}$ Moreso se pregunta “¿Debemos delinear la estructura política de la sociedad de modo que al menos algunos de estos derechos fundamentales sean protegidos por un Bill of Rights?"; y además, "¿Qué rol, si es que alguno, deben tener los órganos jurisdiccionales en la protección de los derechos fundamentales establecidos en el Bill of Rights?"

Obviamente, el debate en juego presupone, entre otras cosas, una difícil discusión filosófica acerca de la "existencia" de ciertos derechos fundamentales. En el marco de este escrito, de todos modos, no voy a ocuparme de tal cuestión, a la que tomaré como supuesta. En lo que sigue, por lo tanto, no analizaré la parte introductoria del artículo de Moreso, dedicada específicamente al tratamiento de una diversidad de problemas conceptuales vinculados con la misma idea de derechos. ${ }^{2}$

Me ocuparé, en cambio, de examinar las respuestas presentadas por el autor frente a preguntas como las arriba citadas. Sucintamente, Moreso sostiene, en primer lugar, que "Si se acepta una teoría de la justicia que contiene principios que establecen derechos fundamentales" luego, tenemos la obligación de diseñar nuestras instituciones políticas de un modo tal que "aumente la probabilidad de obtener decisiones que no 
violen tales derechos fundamentales." El cuadro institucional favorecido en el texto implica la consagración de un "coto vedado" de naturaleza constitucional, destinado a proteger determinados derechos morales. En segundo lugar, Moreso afirma que, aún si el "control judicial de constitucionalidad de las leyes no es un requisito ni necesario ni suficiente para la protección de los derechos fundamentales [...], tampoco hay razones para pensar que sea un procedimiento que deba ser siempre rechazado" (diría más: el autor realiza esfuerzos significativos para demostrar que el control de constitucionalidad puede y merece ser defendido). En las páginas que siguen, me ocuparé de examinar ambas conclusiones, analizándolas separadamente y haciendo referencia a los argumentos presentados por el autor a lo largo de su trabajo.

\section{El "precompromiso" y la justificación del "coto vedado"}

En relación con la afirmación de Moreso acerca de la importancia de contar con un "coto vedado" de derechos (una idea que, en principio, no me resulta inaceptable), conviene presentar algunas observaciones. Ante todo, la principal justificación aportada por el autor para sostener la idea del "coto vedado," que es la de considerar al mismo como un racional "precompromiso," resulta extremadamente débil. Para explicar brevemente el significado de esta estrategia del "precompromiso" conviene apelar (como lo hiciera Jon Elster, quien fue el que se refirió, originalmente, a esta estrategia), a la fábula de Ulises: ${ }^{3}$ se dice que, alarmado ante el riesgo de perder el control de sí mismo (y así también, de su nave), en un futuro próximo, y a causa del canto de las sirenas, Ulises solicitó a sus compañeros de viaje que lo atasen al mástil de la nave. Ulises aceptó, entonces, perder algo de su libertad presente para, en última instancia, incrementar su libertad futura (la coyuntural atadura al mástil lo ayudaba, así, a mantenerse en el control de su empresa). El ejemplo de Ulises ilustraría la racionalidad de la estrategia de la autolimitación, del autopaternalismo. El mismo ejemplo, además, nos permitiría reconocer la racionalidad de las "autolimitaciones" que puede imponerse una cierta sociedad, con el objeto de sortear (como lo hiciera Ulises) previsibles riesgos futuros. Piénsese, por caso, en el riesgo de que una mayoría 
pretenda oprimir a los grupos minoritarios; o en el que aparece frente a un gobernante inclinado a excederse en el ejercicio de sus funciones. Frente a tales situaciones, la sociedad podría -racionalmente- optar por alguna estrategia autolimitativa, como lo sería, típicamente, la de consagrar ciertos derechos fundamentales inviolables (libertad de expresión, respeto de la integridad personal, etc.) que, de ese modo, quedarían protegidos a pesar de los coyunturales cambios de humor o impulsos autoritarios de algunas autoridades públicas. Gracias a ese tipo de limitaciones, ningún gobierno tendría la posibilidad institucional de desarrollar aquellos comportamientos opresivos salvo, por supuesto, a través de un muy costoso procedimiento de revisión constitucional.

Ahora bien, existen múltiples razones para rechazar la pertinencia de una metáfora como la de Ulises, para explicar y justificar ciertos comportamientos sociales, vinculados con el desarrollo constitucional. Ante todo, haría referencia a un problema que podríamos llamar de identidad. La metáfora de Ulises nos habla de un caso de autopaternalismo, en principio, justificado: En el tiempo t1, Ulises decide limitar el rango de acciones que puede realizar Ulises en el tiempo t2, esto es, Ulises se limita a sí mismo. Sin embargo, esta situación tiene poco que ver con la que se presenta en el caso de una sociedad que decide adoptar un texto constitucional. En tal situación, la sociedad S, en el tiempo t1, decide limitarse a sí misma y a las posteriores, en el tiempo t2: la generación que (5, 10 o 200 años atrás) adoptó la Constitución, establece de este modo severos límites sobre las generaciones futuras que ahora, para bien o mal, se encuentran a merced de tal documento, en cuya elaboración no participaron. Más allá de que tal limitación constitucional pueda ser teóricamente aceptable o no, lo cierto es que la violación de tal requisito de identidad (un requisito que es dejado de lado, a menos que se asuma, implausiblemente, que siempre hablamos de una misma y única sociedad, aunque hayan cambiado la totalidad de sus miembros) distorsiona completamente el sentido del ejemplo de Ulises, y la justificabilidad que, en aquel caso, acompañaba al ejercicio del paternalismo. Aunque tenemos razones para pensar que cada uno de nosotros puede limitar legítimamente sus propias acciones futuras, resulta mucho más difícil sostener que una generación puede limitar a las que la siguen. El caso de una sociedad que 
se decide a dictar una Constitución destinada a gobernar a las generaciones futuras no representa un ejemplo de autopaternalismo, como el que aparecía en el caso de Ulises.

Por otra parte, en el caso mencionado, de una sociedad que adopta una Constitución, aparece un problema que podríamos denominar de pluralidad de miembros - un problema, nuevamente, ausente en el caso de Ulises, y que agrega nuevas dificultades a la comparación. En efecto, cuando decimos que una cierta sociedad "dicta" su Constitución, apelamos a convenciones lingüísticas y a formalismos jurídicos que nos autorizan a utilizar tal expresión. Sin embargo, lo que realmente queremos decir, en tal caso ( $y$ a menos que concibamos a la sociedad como un "cuerpo" único, como un "organismo físico") es que una cierta parte de la sociedad, por ejemplo, los convencionales constituyentes, adoptaron una Constitución que (al menos, en la mejor de las hipótesis) ha sido ratificada por todos o por una gran mayoría de los ciudadanos. Este hecho determina una nueva y significativa diferencia en relación con la leyenda griega dado que, en el caso de la sociedad que dicta su Constitución, aparece una trágica posibilidad (ausente en el ejemplo de Ulises): la posibilidad de que la minoría que elaboró la Constitución "ate las manos" del resto de la sociedad, dejando libre las propias (una eventualidad que, según creo, se ha concretado en el curso de la historia constitucional "real" de muchos países occidentales, y que el mismo Elster ha comenzado a admitir, en algunos trabajos recientes).

De lo dicho hasta aquí resulta evidente que, a partir de las profundas diferencias que existen entre el caso de Ulises y las sirenas, y el de la sociedad que dicta su Constitución, el principal argumento presentado por Moreso en defensa del "coto vedado" -el argumento del "precompromiso" - pierde toda significación.

\section{Otros argumentos en defensa del "coto vedado"}

Junto al argumento del "precompromiso," Moreso apela a otras razones, igualmente destinadas a justificar el "coto vedado." Ninguna de 
ellas, sin embargo, parecen contribuir al logro de tal objetivo (ni aún, agregaría, considerando a todos estos argumentos conjuntamente).

En primer lugar, Moreso afirma que el "coto vedado" se justifica debido a que tiende a incrementar la posibilidad de que las decisiones democráticas sean respetuosas de todos los individuos que componen la sociedad. Moreso presenta tal afirmación sosteniendo que a) "es un hecho que las sociedades humanas, en ciertas circunstancias, buscan oprimir a las minorías, negando los derechos individuales de sus miembros;" y que b) "el Bill of Rights constituye un instrumento capaz de interponer obstáculos a aquellas decisiones que violan los derechos de las minorías."

Más adelante, y como forma de sostener la misma idea, Moreso sostiene lo siguiente: a) que la consagración de una carta de derechos puede favorecer, indirectamente, "la racionalidad [de nuestro sistema de toma de decisiones]," puesto que nuestras decisiones resultan habitualmente influidas por "la debilidad de las voluntades concurrentes;" y b) que tales declaraciones de derechos, además, incrementan "la probabilidad de que nuestras decisiones democráticas sean justas" (debido a que ciertas opciones legislativas indebidas resultan precluidas). Finalmente, sostiene Moreso, el "coto vedado" puede defenderse como c) un medio para "proteger la estabilidad de la cultura política fundamental" (la idea, en este caso, es que el "coto vedado" puede garantizar que la cultura política -que abarcaría tanto la separación de poderes, y el sistema federal, como algunos principios fundamentales sobre derechos y deberes- permanezca, de algún modo, aislada "de las presiones de la política cotidiana."

Frente a este cúmulo de argumentos en defensa del "coto vedado," pueden presentarse una multiplicidad de réplicas y objeciones. En primer lugar, y sólo como un detalle formal, deberíamos invitar al autor a realizar una distinción más clara entre sus argumentos, ya que en muchas ocasiones Moreso parece apelar a los mismos de una forma indistinta, o como si existiese una sinonimia entre ellos, lo cual no es en absoluto claro. Hacemos referencia a cuestiones muy diferentes cuando decimos que el "coto vedado" disminuye la posibilidad de oprimir a las minorías; aumenta la racionalidad de nuestras decisiones democráticas; incrementa 
nuestra capacidad de tomar decisiones justas; o favorece la estabilidad de la cultura política. De todos modos, podemos dejar de lado esta cuestión de detalle, y pasar al núcleo de su argumentación.

Las afirmaciones de Moreso suscitan alguna perplejidad no sólo porque se contradicen con la práctica constitucional cotidiana sino además -y lo que es más grave- porque sus argumentos terminan por eludir de un modo sorprendente la cuestión más importante que se proponían resolver (esto es, el problema de la justificación del "coto vedado"). Preguntémonos, por ejemplo ¿es cierto que la adopción de una carta de derechos se encuentra en condiciones de producir la estabilidad? Ello no parece muy obvio. En los Estados Unidos (un país que puede considerarse la "cuna" del Bill of Rights) la institución de los derechos constitucionales fue precedida y seguida de graves conflictos políticos vinculados, en buena medida, con discusiones en torno del Bill of Rights. No resulta un dato menor que el evento tal vez más trágico de la historia norteamericana, la guerra civil, haya tenido que ver con disputas en torno al alcance de los derechos incorporados en la Constitución en donde, implícitamente, se trataba a las personas de color como seres inferiores. Es claro, Moreso podría decirnos que un conflicto como el citado se debió a que los derechos incluidos en la Constitución no eran los correctos. Pero, podríamos replicarle, esto es precisamente lo que sostienen los autores que él critica en su artículo: que no es en absoluto claro cuáles son los derechos que deben ser incluidos en la Constitución. Este es, justamente, el punto que se encuentra en discusión.

¿Y es cierto que la adopción de una declaración de derechos favorece la justicia de las decisiones? Nuevamente, la respuesta positiva o negativa frente a tal pregunta depende de cuestiones coyunturales. Por ejemplo, si, tal como se ha comprobado en la mayor parte de las Constituciones modernas, se parte de (injustificadas) situaciones de profunda desigualdad económica y se inserta una cláusula constitucional de garantía de la propiedad, entonces la declaración de derechos incrementa (en lugar de disminuir) la probabilidad de que se tomen decisiones injustas, opresivas en relación con las minorías (o, directamente, opresivas en relación con las mayorías), e irracionales (en cualquier sentido 
del término). Seguimos ignorando, entonces, por qué es que corresponde defender la adopción de un "coto vedado."

Conviene considerar, además, otras observaciones vinculadas con las afirmaciones de Moreso acerca de la oportunidad de adoptar un "coto vedado." Por un lado (y suponiendo que las Constituciones tienden a sobrevivir a las generaciones que las adoptan), puede sostenerse que, si se adopta una carta de derechos demasiado "robusta," se corre el riesgo de transformarla en un instrumento (no sólo de dudosa justificabilidad, sino además peligrosamente) antidemocrático. En dicho caso, es dable esperar que los ciudadanos de "aquí y ahora" resulten imposibilitados de llevar adelante las discusiones que más les interesan, ya que las discusiones más importantes han sido removidas de la agenda pública. Pero si, en cambio, -y como sugiere Moreso- "los derechos son establecidos [...] de un modo genérico y no detallado," luego se enfrenta el serio riesgo de obtener una Constitución con un contenido aparentemente inobjetado pero que resulte, finalmente, vacuo. ¿Cuál es, en definitiva, el sentido de adoptar una lista de derechos tan abstractos si, en definitiva, ignoramos qué es lo que permiten y qué es lo que impiden?

Si la respuesta a la pregunta anterior, y tal como parece sugerirlo Moreso, es que, de este modo, se favorece una discusión permanente sobre el significado de tales derechos, entonces deberemos decir que el sistema institucional que termina proponiéndonos no resulta, justamente, el que más contribuye a la producción de tal debate (volveré sobre este punto más adelante).

Más todavía: la importancia de la discusión pública sobre los derechos parece estar en contradicción con la estrategia sugerida por Moreso de aislar las cuestiones ligadas a los derechos de las "presiones de la política cotidiana." ¿Es defendible o no, entonces, la posibilidad de que los ciudadanos discutan democráticamente sobre las cuestiones que más les interesan? Moreso parece darnos, al respecto, dos respuestas enfrentadas entre sí. 


\section{La justificación del rol de los jueces en el control de constitucionalidad}

Quisiera agregar algunas breves consideraciones en torno a la segunda de las cuestiones en juego, vinculada al rol de los órganos jurisdiccionales en la protección de los derechos constitucionales (una cuestión a la que Moreso dedica relativamente poco espacio). En sus referencias al control judicial de constitucionalidad, Moreso hace referencia habitual a los escritos del filósofo John Rawls, con el que comparte una curiosa estrategia. En el modesto espacio que dedican al tema del judicial review, ambos se esfuerzan por defender tal opción y criticar posibles alternativas, para concluir sus análisis, sin embargo, con afirmaciones sorprendentemente prudentes (digo "sorprendentemente" porque, en ambos casos, las conclusiones no parecen estar de acuerdo con el tenor de las argumentaciones que las motivaron). ${ }^{4}$

En su breve aproximación al tema, Moreso cita a Dworkin cuando sostiene que "Democracy does not insist on judes having the last word [en las cuestiones de derechos], but it does not insist that they must not have it." ${ }^{5}$ Esta cita constituye uno de los pocos argumentos a los que recurre Moreso para defender el rol de los jueces en el control de constitucionalidad. Ahora bien, más allá de su fuerza retórica y del apoyo que suscita por provenir de un teórico tan lúcido como Dworkin, la cita expuesta nos ofrece un argumento, en verdad, poco interesante, además de muy discutible. De hecho ¿qué es lo que significa que la democracia no requiere que los jueces tengan la última palabra institucional? Si se trata de un juicio descriptivo, según el cual la ciudadanía no realiza movilizaciones públicas en contra del control judicial, ni se moviliza masivamente y con pancartas reclamando que los jueces no tengan la "última palabra" en temas constitucionales, la observación es correcta pero banal. La cuestión que nos interesa es si, en base a una concepción plausible de la democracia, puede denegársele al poder judicial el derecho de decir la última palabra en temas constitucionales.

Personalmente, considero que tenemos todos los motivos para sostener que los jueces no deben tener la última palabra institucional. De todos modos, no voy a ocuparme extensamente del tema, del que me he ocupado ya, con mayor detalle, en otro trabajo. ${ }^{6}$ Simplemente quisiera 
sostener que, si justificamos la democracia, por ejemplo, como un sistema que promueve (o ayuda a promover, mejor que otros sistemas alternativos) la discusión pública, entonces contamos con buenas razones para limitar, aunque no anular, el rol de los jueces en el control de constitucionalidad. Los jueces, sin dudas, pueden contribuir enormemente a la discusión colectiva, por ejemplo, señalándonos hechos y razones que hemos dejado de lado en nuestros debates. Sin embargo, cualquiera que quiera decirnos que, en una democracia, son los jueces y no los ciudadanos o sus representantes los que deben decirnos si, en definitiva, tenemos o no el derecho al aborto, a leer pornografía, a establecer límites sobre la propiedad privada, o a incrementar los impuestos sobre los más ricos, tiene la carga de decirnos por qué: en una democracia, la carga de la prueba la lleva el que intenta ponerle límites a la discusión pública, y no el que defiende su más amplia extensión.

Para concluir, dos breves consideraciones en relación con dos argumentos menos centrales dentro de la estrategia elaborada por Moreso en su (moderada) defensa del control judicial. Por una parte, el autor sostiene que las decisiones judiciales (por así decirlo) "pueden aportarnos más de lo que nos quitan" a nuestra calidad de ciudadanos democráticos. En efecto -agrega- pensemos en qué es lo que puede ocurrir con una ley opresiva: si los jueces anulan dicha norma se consigue, entonces, "un resultado más justo del que se hubiera alcanzado sin la existencia del control judicial" y si, en cambio, los jueces no anulan dicha ley, entonces, "nos encontramos con la misma situación con la que nos encontraríamos sin la existencia del control judicial." Nuevamente, sin embargo, la afirmación de Moreso no nos resulta de gran ayuda. De hecho, podríamos obtener el mismo resultado al que se refiere nuestro autor si, por ejemplo, encargáramos el control constitucional a un buen profesor de filosofía o a un abogado dotado de buenas intenciones. Lo que necesitamos es que Moreso, u otros defensores de la revisión judicial, nos aclaren cuáles son las razones que tenemos para reconocer la validez del particular instrumento escogido (el control judicial), organizado del modo en que hoy lo conocemos.

Finalmente, Moreso nos dice que el procedimiento de control 
judicial no implica que "la constitución sea lo que los jueces dicen que es [dado que] existen procedimientos, aunque costosos, de revisión de la constitución," como existe la posibilidad de "cambiar la composición de la corte, o sus opiniones." Para dar apoyo a tales dichos Moreso agrega, citando a Rawls, que "The constitution is not what the Court says it is. Rather, it is what the people acting constitutionally through the other branches eventually allow the Court to say it is." ${ }^{\text {" }}$ Nuevamente, nos enfrentamos a una serie de consideraciones más relevantes por su fuerza retórica y por la notable fuente de la que provienen, que por el valor de su contenido. Por más que filósofos de la talla de Rawls (o, sin dudas, el propio Moreso), nos digan que la Constitución es aquello que nosotros le autorizamos a la Corte que diga, lo cierto es que (al menos, en sistemas judiciales como el nuestro) el significado último de la Constitución sigue dependiendo del criterio de los jueces, aunque ello no nos guste, o nos obstinemos a decir lo contrario, u organicemos manifestaciones para inducir a los jueces a que adopten tal o cual decisión. Los españoles tienen o no el derecho al aborto, en última instancia, de acuerdo con lo que digan sus jueces. Los norteamericanos tienen o no el derecho a penalizar la homosexualidad, en última instancia, conforme a cuál es la opinión de los jueces. Hechos tales como que, por ejemplo, el presidente Roosvelt haya amenazado con modificar la composición de la Corte, luego de una serie de fallos adversos a sus políticas; o que el presidente Menem en la Argentina haya aumentado el número de jueces del máximo tribunal para obtener una mayoría favorable, no nos hablan de la bondad de nuestro sistema institucional, sino de su tremenda rigidez; no nos habla del modo en que el "pueblo" influye en las decisiones de los tribunales, sino de las enormes dificultades que el "pueblo" encuentra para ejercitar su influencia.

Con este tipo de afirmaciones, obviamente, no quiero sostener que la ciudadanía deba disponer de una ilimitada facultad para llevar adelante su propia voluntad. Me interesa decir, simplemente, que si se establecen límites sobre dicha voluntad (como debe hacerse), entonces corresponde que justifiquemos el tipo de controles que adoptamos, y que demos razones de por qué le permitimos a tales personas y no a otras el realizar tales tareas de supervisión. En este sentido, el modo en que actualmente 
se ejerce el control de las leyes (y que Moreso, en principio, defiende) merece recibir razonables y radicales críticas por parte del ciudadano animado por una vocación democrática. Llegados a este punto, de todos modos, no corresponde otra cosa que el agradecer la lucidez de Moreso, y agradecer también el estímulo que recibimos a partir de sus trabajos, para continuar con nuestras discusiones sobre la Constitución. 


\section{Notas}

1 En parte, la "resurrección" de la que hablo se vincula con la reciente publicación de dos trabajos relevantes dentro de la discusión citada En primer lugar, el escrito de Ronald Dworkin, 'Does Britain Needs a Bill of Rights?' en Ronald Dworkin, Freedom's Law. The Moral Reading of American Constituion, Oxford University Press, Oxford, 1996, pp. 352372; y la respuesta de J. Waldron, 'A Right Based Critique of Constitutional Rights', en Oxford Journal of Legal Studies, 13 (1993), pp. 18-51.

2 En esta primera parte (que, según entiendo, no era estrictamente necesaria para la argumentación que desarrolla a continuación), Moreso se ocupa de tres cuestiones principales: a) demostrar que la idea de derechos que toma en consideración es "metaéticamente neutral;" b) demostrar que "la tesis de los derechos es (parcialmente) neutral desde el punto de vista de la ética normativa;" y c) establecer algunas precisiones en torno al concepto de derechos morales.

${ }^{3}$ Ver, Jon Elster, Ulysses and the Syrens, Cambridge University Press, Cambridge, 1988.

${ }^{4}$ Rawls concluye afirmando: "no he tratado de defender [la revisión judicial de las leyes], aunque podría defenderse [tal práctica] dadas ciertas circunstancias históricas y ciertas condiciones de la cultura política." John Rawls, Political Liberalism, Columbia University Press, New York, 1993, cap. 6.6. Según Moreso: "que el control judicial de constitucionalidad sea un instrumento adecuado para defender el "coto vedado" de los derechos depende de consideraciones contingentes y estratégicas," y además: "la conveniencia de los mecanismos de control judicial de la constitucionalidad depende [...] de circunstancias históricas y contingentes."

${ }^{5}$ Ronald Dworkin, 'Introduction: The Moral Reading and the Majoritarian Premise', en Ronald Dworkin, Freedom's Law, op.cit., p. 7.

${ }^{6}$ Roberto Gargarella, La justicia frente al gobierno, Ariel, Barcelona, 1996.

${ }^{7}$ John Rawls, Political Liberalism, op. cit., p. 237. 